\title{
E-Government Applications for Integrated Access to Complex Data Resources Using Multi-Agent Systems
}

\author{
Roumen Trifonov ${ }^{1}$ and Radoslav Yoshinov ${ }^{2}$ \\ ${ }_{1}^{1}$ Department Computer Systems at Technical University - Sofia, 1000 Sofia, Bulgaria \\ 2 Telematics Laboratory at Bulgarian Academy of Sciences, 1000 Sofia, Bulgaria
}

\begin{abstract}
Currently the main focus of e-Government in Bulgaria is aimed at the provision of electronic administrative services to citizens and businesses. The development aimed at streamlining and optimizing the work of administration and information support of processes of decision-making is still unregulated. They are not conducted any serious research. The paper points out one of the activities which according to the authors may fruitfully contribute to the development in this direction. It is about an integral approach to access to complex information resources (databases), supported by different systems, including in different countries. For realization of this approach the authors propose the application of multi-agent systems in an environment of semantic Web services.
\end{abstract}

\section{Introduction}

According to the latest strategy of the Bulgarian government: "E-governance is the transformation of state structures through the use of information and communication technologies, entirely geared towards consumers. It is done in parallel with organizational changes and the acquisition of new knowledge to improve public services and democratic processes and the creation and implementation of state policy."

Actually, the main aspect of e-Government in Bulgaria is focused to the provision of electronic administrative services to citizens and businesses. The development aimed at streamlining and optimizing the work of administration and information support of processes of decision-making is still unregulated. They are not conducted any serious research.

At the same time, the experience of some advanced countries [1] [2] shows that automation of the processes of decision-making in administrative structures has potential not less than the services in order to enhance the public effectiveness. By the way, while in executive agencies and municipal administration the services for citizens and businesses occupy a significant place, in the activities of the ministries these functions almost absent.

The observations of the authors in the process of collaboration with the administrative authorities (particularly at the central level) allow the conclusion that perhaps the most fruitful direction in the automation of the process of decisions making by administrative authorities would be the organization of effective integration of data from heterogeneous information sources, organized by various technologies and various target functions [3].
Proceeding from these circumstances, the present article attempts to indicate one of the possible directions for informational support of decision making in administration. On the base of international experience, the authors suppose that this may be a combination of two technologies. On the one hand, the application of so called Semantic Web technologies. On the other hand, as management tool for the environment of Semantic Web services the present article proposes to use system of autonomous intelligent agents.

\section{The basis of the proposed approach}

Intelligent agents and semantic web services are two technologies with great potential. Striking new applications can be developed by using the tools and techniques they provide. However, Semantic Web Services need for an upper software entity able to deal with them and, on the other hand agent technology has historically suffered from a number of drawbacks that must be addressed. Integrating these two technologies in a joint environment can overcome their problems while strengthening their advantages [4].

The Semantic Web aims at adding semantics to the data published on the Web, so that machines are able to process these data in a similar way a human can do. For this, ontologies are the backbone technology.

Traditionally, the Web has been conceived as a distributed source of information. The emergence of Web Service Technology has permitted to extend it to a distributed source of functionality. Web Services make applications available on the Web in a standard way so that they can be accessed regardless of the operating

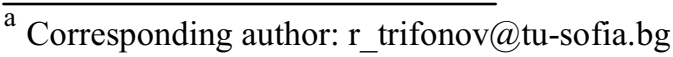


system they are deployed on and the programming language they are implemented in.

The joint application of Semantic Web and Web Services in order to create intelligent Web Services is referred to as Semantic Web Services. Semantic Web Services consist of describing Web Services with semantic content so that service discovery, composition and invocation can be done automatically by, for example, the use of intelligent agents able to process the semantic information provided. The W3Consortium is currently examining various approaches with the purpose of reaching a standard for the Semantic Web Services technology. The recently proposed $\mathrm{W} 3 \mathrm{C}$ recommendation SAWSDL (Semantic Annotations for WSDL and XML Schema [5] identifies some WSDL and XML Schema extension attributes that support the semantic description of WSDL components.

The Intelligent Agent (IA) and Multi-Agent System (MAS) area has received ever-increasing attention by researchers over the last few years. This field emerged due to the promising benefits of having applications with a technology that allows systems to decide for themselves what they need to do in order to satisfy their design objectives. A common accepted definition of the term 'Agent' determines that an agent is a computer system situated in some environment and capable of autonomous action in this environment in order to meet its design objectives [6]. It is necessary to highlight that an agent has to fulfil some properties in order to become intelligent: reactivity (i.e. the ability to perceive its environment and respond to changes in it in a timely fashion), pro-activeness (i.e. the ability to exhibit goaldirected behaviour by taking the initiative), and social ability (i.e. the ability to interact with other agents).

During the process of transforming web services into agents or boxing them into agents, the web service description may be retrieved automatically by examining its description in WSDL (web service description language). Converted agent description can be extended with specific ontologies. Agents can be connected to web services by using a gateway agent which keeps two directories - one that serves the agent world and another that serves the web service world.

\section{The functioning of the proposed approach}

There is currently a great quantity of independent and heterogeneous data resources, accessible through the Internet, covering information, which can be useful for administration in order to decision making. Most of this information is stored in databases, although in the last years some ontologies have been developed to harmonise the terminology used in the different databases and to promote integrated access and interoperability among different information resources and applications.

The increasing number of independent and heterogeneous databases produced high inefficiency when the developers wanted to search for information across different databases. So, finding relevant information was rather difficult due to the terminological diversity. Given this situation, different entities that had developed their own databases often work together to define a common vocabulary for modelling how terms might be annotated.

Despite their ability to provide a high degree of interoperability across platforms and operating systems, Semantic Web Services infrastructures possess neither enough degree of autonomy nor ability to automatically adapt to changing situations. In these settings, agents can contribute to make systems more autonomous and dynamic, thus maximising their perceived utility. In line with this, the approach presented here comprises both Intelligent Agents and Semantic Web Services working cooperatively in the same environment. Ontologies act as the facilitating technology thus making it possible to exploit the advantages these technologies offer separately. This solution stems from a basic underlying hypothesis: Intelligent Agents and Semantic Web Services were conceived for quite different purposes, and so, they must lie on different abstraction layers. The main idea behind Agent Technology was not for Intelligent Agents to be able to provide services, but to act as autonomous entities that incorporate intelligence and cognitive capacities, which allow them to show a goaloriented pro-active behaviour and to establish, either competitive or cooperative, interaction processes with other software entities in order to satisfy their design objectives. On the other hand, Web Services involved a further evolution in the distributed computing field and their only purpose is to provide accessible functionality.

These conceptual differences between Agent Technology and Semantic Web Services Technologies lead to the need to have both technologies working in an integrated environment and glimpse the advantages of their combination in the development of complex systems. Ontologies [7] are the paramount technology in the presented approach as they operate as the 'glue' that binds the remaining components of the architecture.

The ontologies function as universal vocabularies so that Web Services and agents share the same interpretation of the terms contained in the messages that they exchange. From the Web Services point of view, ontologies are useful to semantically describe Web Services' capabilities and processes.

This semantic description can then be automatically processed by software entities, so that Web Service discovery, composition, selection, execution and monitoring can be done without human intervention. On the other hand, from the perspective of the agents, the local domain-related knowledge of each agent may be extracted from, or built upon, the application domain ontology. Moreover, inter-agent communication may be carried out by means of a common vocabulary based on an agreed ontology.

The negotiation processes between agents may take place in accordance with protocols represented in an ontology and by using the related strategies also stored in an ontology.

The practical implementation of the proposed approach has as main objective to develop an agent-based knowledge manipulation framework. One of possible 
architectures of this framework can consist of following modules (Fig. 1):

- ontology design component with user friendly interface;

- agent-based Web Service search module;

- knowledge Database (KDB);

- Semantic Web Service composition module;

- user interface for displaying the results.

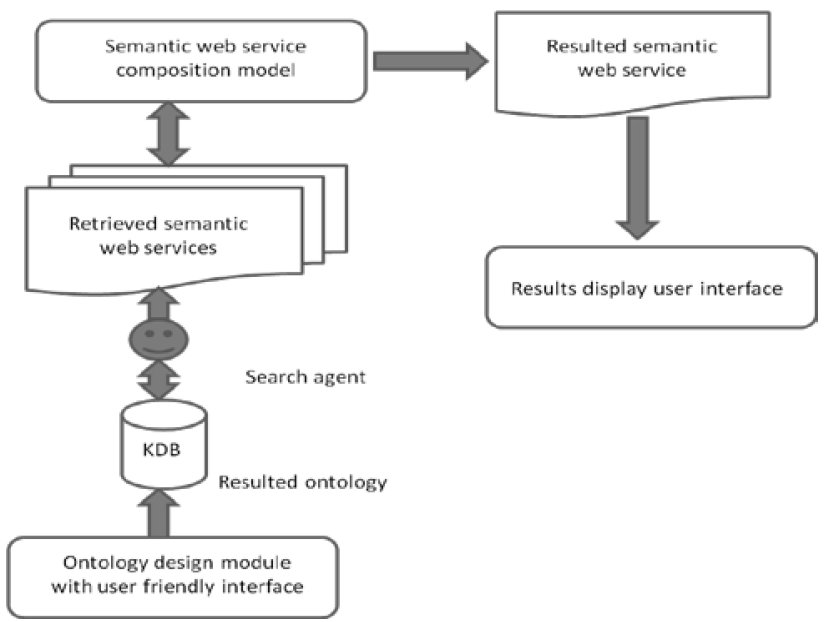

Figure 1. Architecture of agent-based knowledge manipulation framework

In order for intelligent agents to successfully carry out their assigned tasks, those ones must have access to various data repositories containing the knowledge that is necessary to fulfil the assignment. These repositories can be either local or external to the system. As data repositories, four kinds of ontologies have been included within the platform:

1. Application and domain ontology - the application ontology contains the knowledge entities (i.e. concepts, attributes, relationships, and axioms) that model the application in which the framework is to be employed. On the other hand, the domain ontology, represents a conceptualisation of the specific domain the framework is going to be applied in. This ontology supports the communication among the components in the framework without misinterpretations.

2. Agent local knowledge ontology - it contains, for each agent, the knowledge about the environment it possesses. This ontology generally includes knowledge about the assigned tasks, as well as the mechanisms and resources available to achieve those tasks.

3. Negotiation ontology - it comprises both negotiation protocols and negotiation strategies that constitute the negotiation mechanisms agents must use to coordinate their interactions. With this ontology, agents can choose the best mechanism to use for coordinating their actions, which highly depends on the problem under question and the application domain.

4. Semantic Web Services ontologies - in this repository (that can be comprised of various ontologies distributed all over the Internet) the ontologies that contain the semantic description of Web Services are stored. The framework does not impose any restriction in terms of the kind of Semantic Web Services specification to be used.

\section{Conclusions}

The objective of the present paper is to contribute for overcoming the unilaterally understanding of eGovernment, limited to electronic administrative services for citizens and business.

It should be borne in mind that there is a fundamental difference in the computing processes that underlie these activities. While the provision of services can be attributed to a category "Real Time Information Processing" and there are a pre-domination of formalized procedures, structured information and mode kindred to real-time; the decision-making process belongs to the category "Knowledge Management". There are a predomination of analytical and synthetic procedures, unstructured information, non-formalized data and nonregulated time periods for solutions generation.

As about the semantic interoperability of processes, the service delivery uses deterministic processes based on registers, performing the role of "data clearinghouse" and semantic filters, the role of which is performed by UEEDE. In the case of decisions making the semantic interoperability is based on a stochastic approach.

This determines differences in the instruments for implementation of these two types of activities.

\section{References}

1. The Role of Business Intelligence in Government: A Case Study of Swedish Municipality Contact Centers, Trollhattan (March 2010)

2. e-Government Intelligence "Ca' Foscari” University of Venice (2010)

3. United Nations E-Government Survey 2014 EGovernment for the Future We Want, New York, (2014), 264 p., ISBN: 978-92-1-123198-4

4. Shalini.L, Femila Goldy.R Efficient Discovery of Semantic Web Services, International Journal of Innovative Research in Science, Engineering and Technology Volume 3, Special Issue 3, (March 2014) ; ISSN: 2319 - 8753

5. Semantic Annotations for WSDL and XML Schema W3C Recommendation (28 August 2007)

6. Agent Technology: Enabling Next Generation Computing A Roadmap for Agent Based Computing AgentLink (2003)

7. Web Service Modeling Ontology (WSMO) W3C Member Submission (3 June 2005) 